\title{
Preface: Pregnancy in GI-disorders
}

Planning pregnancy, being pregnant and becoming parents is an important phase in life. Pre-existing chronic gastrointestinal or liver diseases affect women in their young and reproductive years of life and the chronic character means that lifelong medical treatment is often required. Anxieties on many aspects of the disease management in this vulnarable period such as the effect of pregnancy on disease, the effect of the drugs and disease on the fetus and the potential of passing of the disease to offspring are affecting choices made by parents to be. A careful consultation on the effects of maintenance drugs on fertility, disease remission during conception and pregnancy and the outcome of their children is justified for maintaining disease remission and involves adequate preconception counselling as explained in this special issue on pregnancy.

Further in this issue of Best Practice and Research: Clinical Gastroenterology up to date reviews by outstanding authors are provided on several aspects of managing patients with a preexisting gastrointestinal or liver disease in pregnancy. As understanding of the immunological changes that take place during pregnancy may aid in counselling patients with a pregnancy wish several aspects on immune tolerance in and outside the uterus, effect of hormones and the microbiome are discussed in an excellent chapter on the immune system and microbiome and pregnancy. The interpretation of liver function tests in pregnancy can be rather challenging, liver tests abnormalities occur in $3-5 \%$ of pregnancies and can have many different causes. Liver disease unique to pregnancy are: intrahepatic cholestasis of pregnancy, acute fatty liver of pregnancy and HELLP syndrome. But also pre-existing chronic liver diseases could influence liver tests during pregnancy and more importantly careful balancing the use of drugs, the effect of pregnancy on the disease, and on transmission of liver diseases require early management of pregnancy wish.

For inflammatory bowel disease the introduction of biologics has revolutionized the treatment of this chronic and often invalidating disease. However, knowledge of the effects of these drugs on the fetus is at launch of these drugs always rather limited. As many of these biologics are actively transferred through the placenta questions arise to minimize fetal exposure. During pregnancy there are a number of physiological changes that could alter pharmacokinetics and pharmacodynamics and intriguingly this phenome could be used for a personalized biologic pregnancy plan. Current knowledge on placenta transfer and the effects and safety on adjusting biologic treatment in pregnancy are discussed in a review summarizing available data on through levels during pregnancy. Also, authoritative reviews are provided on the role of imaging and surgery during pregnancy.

A big thank you to all of the authors for taking time out of their busy schedules to contribute to these chapters. It was a real privilege to edit this issue on pregnancy, and I expect that it will lead to an optimized pregnancy care pathway for our patients.

C.J. Van der Woude

Department of Gastroenterology and Hepatology, Erasmus Medical Center, Rotterdam, the Netherlands 